\title{
Research on Dynamic Scheduling of Automatic Rail-guided Vehicle
}

\author{
Zhixing Song \\ School of North China Electric Power University, Baoding 071000, China. \\ 504115681@qq.com
}

Keywords: RGV; Priority; Particle swarm optimization algorithm; Exhaustive method.

Abstract: With the rapid development of artificial intelligence, intelligent processing systems have slowly entered our lives. The control of intelligent machines through computers can improve the efficiency of work. The RGV is an unmanned, smart car that can run freely on a fixed track. According to the working conditions of RGV, the corresponding model is established, and three sets of data are given for specific analysis and calculation.

Step 1: build a model. The analysis shows that the factors affecting the efficiency of the processing system are related to the distance of the RGV from the CNC that sends out the signal, the loading and unloading time on different sides, the number and position of different blades, and the difference in working time between different processes. Define the priority $\mathrm{K}=\mathrm{Mi}+\mathrm{C}+\mathrm{D}$, and determine the service sequence of RGV by the size of $\mathrm{K}$. Taking the time as the standard, the priority Mi of different distances and the priority $\mathrm{C}$ of the two sides of the loading and unloading time are obtained, and the priority $\mathrm{D}$ of the working hours of the two processes is obtained by the particle swarm algorithm. By optimizing the exhaustive method for all the solutions of the tool arrangement, the optimal tool arrangement is obtained.

Step 2: Test the model. Three sets of data were brought into the model to test the validity of the model. Set the standard of the inspection model. If the number of processed materials obtained by using the scheduling strategy is larger than the number of materials obtained by the original scheduling scheme, it can be concluded that the model is valid.

This model takes into account the distance between the RGV and the CNC that sends the signal, the time of different processes, the number of different blades, which greatly increases the processing efficiency of the system. Particle swarm algorithm and exhaustive method improve the optimality of dynamic scheduling scheme.

\section{The composition of intelligent processing systems}

\subsection{Scene description}

Figure 1 is a schematic diagram of an intelligent machining system consisting of 8 computer numerical control machine (CNC) and 1 Rail Guide Vehicle (RGV), 1 RGV linear track, 1 feeding conveyor and a conveyor belt. The RGV is an unmanned, smart car that can run freely on a fixed 
track. It can automatically control the moving direction and distance according to the command, and comes with a robot arm and two mechanical grippers. It can complete the loading and unloading operation at the same time, and also has a material cleaning tank to complete the cleaning material task.

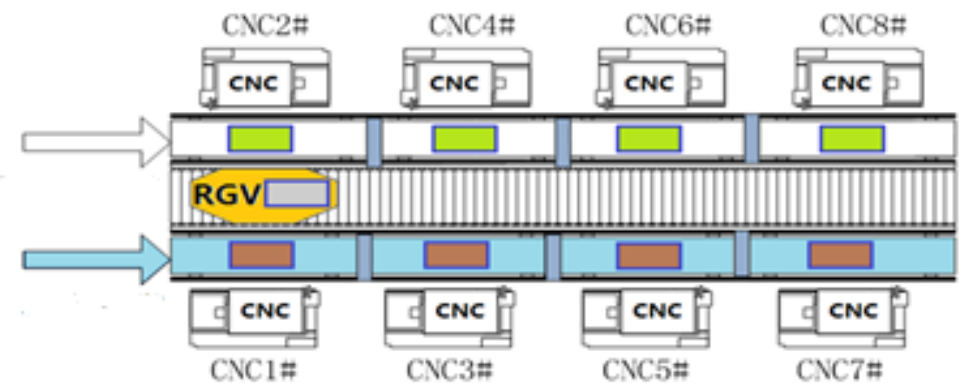

Figure 1 the schematic diagram of an intelligent machining system

\subsection{System composition and operation process}

(1) CNC: Install 4 CNCs on both sides of the loading conveyor and the unloading conveyor, equidistantly arranged.

(2) RGV: RGV has an intelligent control function that can receive and send command signals. According to the command, it can move on the linear track and stop waiting. RGV can only perform one of moving, stopping waiting, loading and unloading, and cleaning at the same time.

(3) The time required for the RGV to be an even numbered CNC to be loaded and unloaded at one time is greater than the time required for the odd-numbered CNC to load and unload at one time.

(4) After the RGV completes the loading and unloading operation for a CNC, the robot arm is rotated, and the clinker of one robot is moved to the top of the cleaning tank for cleaning operation (only the processed clinker is cleaned).

(5) Work 8 hours per shift.

(6) After the intelligent processing system is powered on, the RGV is in the initial position between CNC1\# and CNC2\#, and all CNCs are in the idle state.

\section{Symbol Description}

Table 1symbols description

\begin{tabular}{c|c}
\hline symbols & Explanation \\
\hline $\mathrm{K}$ & priority \\
$\mathrm{M}$ & $\begin{array}{c}\text { The distance priority of the CNC that } \\
\text { sends the demand signal to the RGV }\end{array}$ \\
\hline $\mathrm{C}$ & Priority of loading and unloading time \\
\hline $\mathrm{D}$ & Priority of different process machines \\
\hline $\mathrm{t} 1 \mathrm{t} 2 \mathrm{t} 3$ & Move one, two, three units of time \\
\hline td & $\begin{array}{c}\text { Loading and unloading time of the lower } \\
\mathrm{CNC}\end{array}$ \\
\hline tu & $\begin{array}{c}\text { Upper and lower CNC loading and } \\
\text { unloading time }\end{array}$ \\
\hline
\end{tabular}




\section{Building a model}

\subsection{Analysis of influencing factors}

Analysis system workflow can be concluded that the factors affecting RGV work efficiency are: the distance of the CNC sending the demand signal, the difference of the loading and unloading time, the number of the two blades and the location of the installation, and the difference between the time required for the two processes. The best solution for blade placement needs to be calculated.

It is easy to conclude that the closer the CNC is to the RGV, the higher the priority of the CNC with less loading and unloading time. That is, if the distance is the same, the $\mathrm{CNC}$ on the lower side of the service is preferentially served; when the demand signal is sent on the same side, the CNC with a short service distance is prioritized.

How to determine the RGV's movement when performing the first process (denoted as machine one) and the CNC (recorded as machine two) performing the second process simultaneously. Assume that when the machine is first processed, the material of the machine 1 is first removed, and then the new material is placed. In the second step, the material of the machine 2 is removed, and the processed material of the machine is placed on the machine 2, the third step, The processed material is washed and placed on the unloading conveyor. If the machine 2 is processed first, the material of the machine 2 is first removed, and then the cleaning is performed. After the cleaning is completed, the material on the machine is removed and placed on the machine 2. Compared with the two processes, the second process has one more time for loading and unloading, and the machine waits for one cleaning time. Therefore, it is concluded that the CNC performing the first process should be prioritized when the two CNCs simultaneously emit signals without considering other factors.

\subsection{Modeling}

Through the above analysis, the priority formula can be obtained as:

$$
K=M i+C+D
$$

Where Mi indicates the priority when the unit is i units, $\mathrm{C}$ indicates the priority of the loading and unloading time, and D indicates the priority of the CNC with the different blades installed. Assume that the time to move one unit is t 1 , the time in two units is $t 2$, and the time in three units is $\mathrm{t} 3$. Assume that the priority when not moving is 0 . The smaller the size of the number, the higher the priority, you can get:

$$
M i=\frac{t i}{t 1}(i=1,2,3)
$$

Assume that the loading and unloading time of the lower CNC is td, and the loading and unloading time of the upper $\mathrm{CNC}$ is tu:

$$
T=\frac{t d-t u}{t 1}
$$

Since considering D, not only the time of loading and unloading, but also the waiting time of a certain machine, we cannot simply derive the value of $\mathrm{D}$ based on the time relationship. To determine the size of $\mathrm{D}$, a particle swarm algorithm is used. According to the formula, it can be 
concluded that the proportions of the loading and unloading time are $\frac{t d}{t 1} 、 \frac{t u}{t 1}$, and then compare their size to get the range:

$$
\left[\left(\frac{t d}{t 1}, \frac{t u}{t 1}\right) \min \times 0.1,\left(\frac{t d}{t 1}, \frac{t u}{t 1}\right) \max \times 10\right]
$$

The above formula is the solution space of the particle swarm algorithm, and then seeks the optimal solution $\mathrm{D}$ of the algorithm through iteration.

The formula for the priority K can be derived:

$$
K=\frac{t i}{t 1}+\frac{t d-t u}{t 1}+D(i=1,2,3)
$$

Based on this, a series of different priority matrices for the position of the RGV can be obtained. For example, the blade on the upper side is placed in the second process, and the lower side is placed as the blade in the first process, and the RGV is located at the CNC8\#. In this case, the matrix of $\mathrm{K}$ is:

$$
\left[\begin{array}{cccc}
M 3+C+D & M 2+C+D & M 1+C+D & D \\
M 3 & M 2 & M 1 & 0
\end{array}\right]
$$

With the priority criteria, we need to design the number of blades and the location of the installation. Assuming that the optimal blade placement is set at this time, the number of processed materials can be calculated according to the priority rule. The number of processed materials can be used as a way to judge whether the blade arrangement is good. By violent search method, the 28-2 blade placement schemes (obviously not all of the same blades) can be searched, and the arrangement with the largest number of processed materials can be obtained. This scheme is the optimal solution for the blade arrangement, and then the optimal scheduling strategy is obtained.

\section{Model checking}

\subsection{Analysis}

First define a standard: the order in which the CNC issues the request is the order of the RGV jobs, and the RGV only serves the CNC in this order in the service process. The arrangement of the blades is to install the first process blade for all the four CNCs on the lower side, and the second process blade for the CNC on the upper side. If the number of processed materials calculated by RGV after adding the strategy is greater than the number of materials obtained under this scheme, it can be verified that the model is valid.

For the scheduling strategy, variables are defined in the algorithm to record the CNC number of the service during the RGV movement process, and a series of changes of the variables are read to obtain the dynamic scheduling process of the RGV.

When using the particle swarm algorithm to calculate the value of the priority $\mathrm{D}$, it is found that the calculation time is too long, and then the algorithm is optimized. We take 5 particles for optimization. Although the calculation time is shortened a bit, if you search once for the data, it is a waste of time. The reason for the analysis may be due to the excessively long time caused by the nested exhaustive method in the particle swarm optimization algorithm. Finally, it is decided to divide the solution space by 0.01 unit length, and use the exhaustive method to roughly search for the value of $\mathrm{D}$. Through the test of three sets of data, this method is basically the same as the result 
calculated by the particle swarm algorithm.

\subsection{Substituting data}

In order to test the practicability and effectiveness of the model, three sets of system operating parameters were given, as shown in the following table 2 .

Table 2 system operating parameters

\begin{tabular}{c|c|c|c}
\hline System operating parameters & Group 1 & Group 2 & Group 3 \\
\hline Time that the RGV move 1 unit taking & 20 & 23 & 18 \\
\hline Time that the RGV move 2 unit taking & 33 & 41 & 32 \\
\hline Time that the RGV move 3 unit taking & 46 & 59 & 46 \\
\hline $\begin{array}{c}\text { The time required for CNC machining to complete } \\
\text { the first process }\end{array}$ & 400 & 280 & 455 \\
\hline $\begin{array}{c}\text { The time required for CNC to complete the second } \\
\text { process }\end{array}$ & 378 & 500 & 182 \\
\hline $\begin{array}{c}\text { Time that RGV load and unload a material for } \\
\text { CNC1\#, 3\#, 5\#, 7\# at one time taking }\end{array}$ & 28 & 30 & 27 \\
\hline $\begin{array}{c}\text { Time that RGV load and unload a material for } \\
\text { CNC2\#, 4\#, 6\#,8\# at one time taking }\end{array}$ & 31 & 35 & 32 \\
\hline $\begin{array}{c}\text { Time that RGV completes a cleaning operation of a } \\
\text { material taking }\end{array}$ & 25 & 30 & 25 \\
\hline
\end{tabular}

Bring three sets of data into the model, you can get the $\mathrm{D}$ value corresponding to data one, data two, and data three, and the blade placement scheme is as shown in the table3.

Table 3 the blade placement scheme

\begin{tabular}{|c|c|c|}
\hline & $\begin{array}{c}\text { Optimal blade } \\
\text { placement }\end{array}$ & Optimal D value \\
\hline $\begin{array}{c}\text { First set of } \\
\text { data }\end{array}$ & {$\left[\begin{array}{llllllll}2 & 1 & 1 & 2 & 1 & 2 & 1 & 2\end{array}\right]$} & 1.12 \\
\hline $\begin{array}{c}\text { Second set of } \\
\text { data }\end{array}$ & {$\left[\begin{array}{llllllll}2 & 1 & 2 & 1 & 2 & 1 & 2 & 1\end{array}\right]$} & 1.04 \\
\hline $\begin{array}{c}\text { Third set of } \\
\text { data }\end{array}$ & {$\left[\begin{array}{llllllll}1 & 2 & 2 & 1 & 2 & 1 & 1 & 1\end{array}\right]$} & 1.20 \\
\hline
\end{tabular}

Explanation of the placement of the optimum blade in the table: 1 indicates the blade in which the first process is placed, 2 indicates the blade in the second process, and the order of the array indicates the type of blade placed from CNC No. 1 to No. 8.

Observing the dynamic scheduling sequence of the three sets of data, we found that the scheduling order of RGV changes very obviously. Three situations are shown here, as shown in Figure 2, Figure 3, and Figure 4.

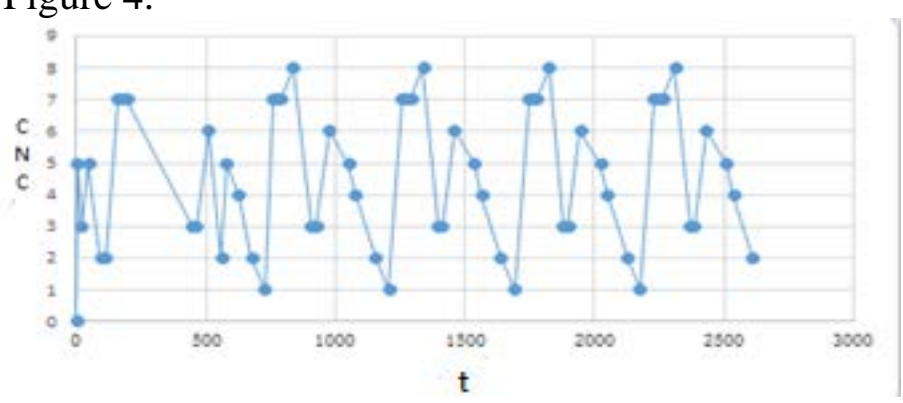

Figure 2 situation of the first set of data 


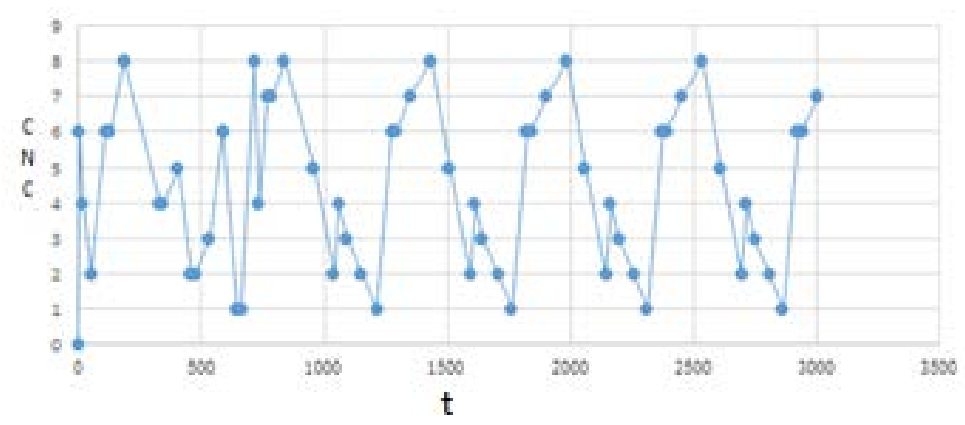

Figure 3 situation of the second set of data

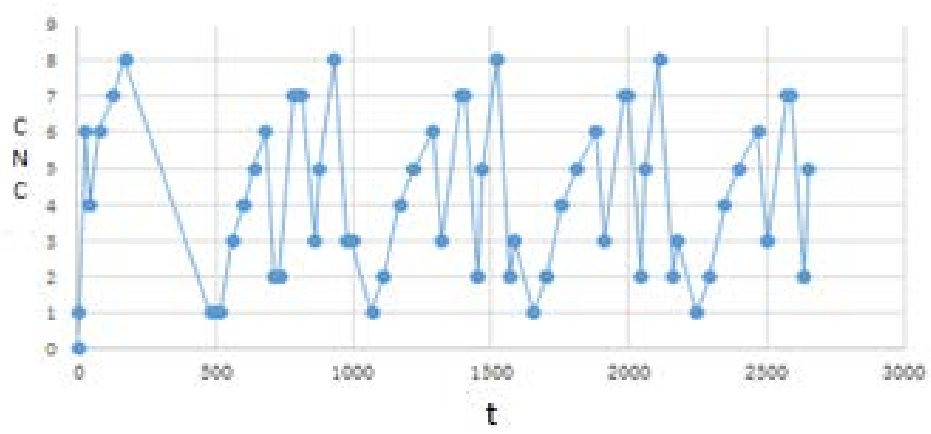

Figure 4 situation of the third set of data

From the figure, we can see that the time of work of the two processes is an important factor affecting the dynamic scheduling change of RGV. The number of materials processed by the three sets of data of the two scheduling schemes is counted, and the figure 5 is obtained.

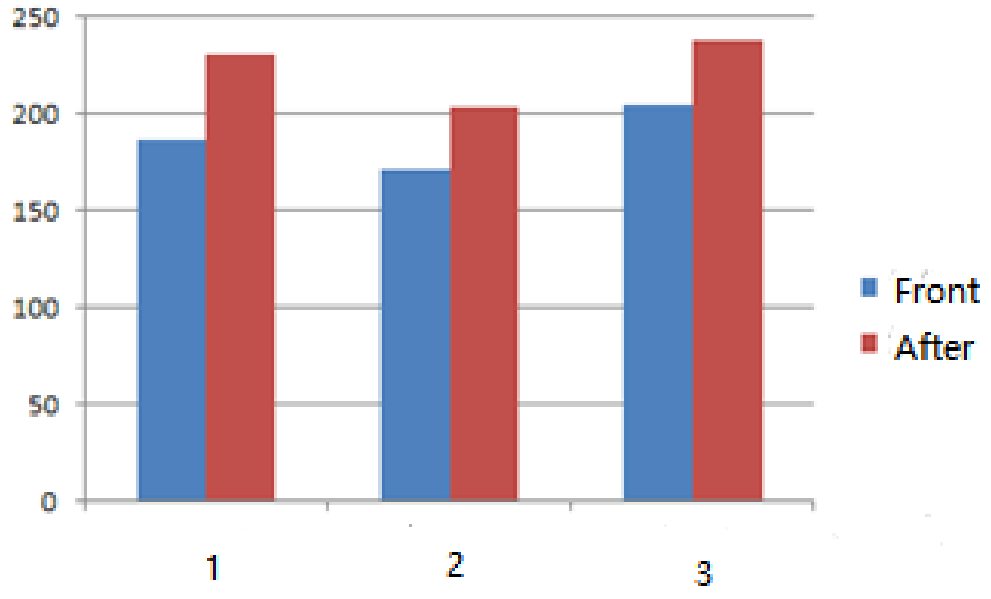

Figure 5 dynamic scheduling changes in two scheduling schemes

It can be seen from the figure that under the dynamic scheduling considering the priority, the number of processed materials in the same time is obviously increased, which proves that the model is very effective.

\section{Conclusion}

After considering the influence of the distance between the RGV and the corresponding CNC and the loading and unloading time, the number of different blades and the position arrangement on the efficiency of the system work, the model is established. Then according to the change of 
working parameters, the particle swarm algorithm and the exhaustive method are used to calculate the priority value and the placement of the blade, which has strong applicability. In the particle swarm optimization algorithm with nested exhaustive methods, the optimization time will be longer, and there may be better ways to find the best.

\section{References}

[1] Information on: http://www.mcm.edu.cn/html_cn/node/7cec7725b9a0ea07b4dfd175e8042c33.html 